\title{
NIVELES Y DIMENSIONES EN LA EXPLICITACIÓN HUSSERLIANA DEL HORIZONTE HISTÓRICO
}

Roberto J. Walton*

StNTRSE - Bn atru escrito "A ccipem da peametrin", Hueseel trota do bocizocto, ds hieturia cuji estrumura dsve ser invest/gads motodiosmentse "A explictisça do bcrizcnte, que peceocrences. nho deva permanecer apenas num discurao vago - supertectal, mas deve alcasçat um tipo de

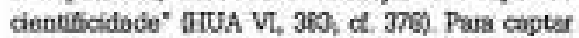
a indole peculis do hortzocts bistitico dew-se bevar en coenta primeiramente a conceite de "pre-darse' (Vongogobenhar). Uma dupls inter.

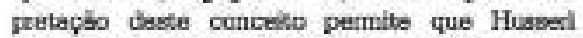
examine a histiria como possiblidade e a histhtia como efetividste. Isto significa que, atroves das maltiplas realidades que a hiatcola pos clerece sesain cosdjọbes de poseiblildade para que poesamos tor un berlannte histirica. Aldm dianq. tems relacilo com a paserbilifaris tants quanto com a eletiridade, 6 posstvel tracar duse distinçôes ulteriares. Ascim, pode-se distinguir quatzo tiveis diferentes da interpostaçào da histd́cia: 1) o rivel des condiçoes de peesihilidade, teso 4, da implicapio dos cutras, o tempo e o mundo; 210 rivel dss condiçes drévias pars a efeclvaçăo, sto 6, da "protogeratividade", a tradicsonalizaçĭo

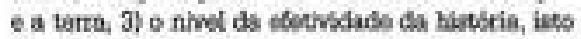
s. de ura forma de peraclat ume tradticla e um tecritorio e 4) o nivel da gececatividede espeitual socicnal, de "uma" trodiçio e de tepresuntaçẩ de "um" mundo. O cerminho da investigaça
Hestaio - En au escrito -Ei coigen de la geametria", Huaven eacribe acber ef batiocnte de la historh, cuya ertructusa debe ser invertionda Eatobdicamente. "La expilictacike det herizonte, a la gue vecurnimce, no debe permanscer en un decurso wago $y$ superticial, sino que debe ella misms alcaneat un tipo de clentisidad" (Hua VI, 383: ㄷ. 3780. A fin de captar la indoin peculiat Sel boriwnte histocico, se debe tecuet en cuemta en pamer lugar al poccepto de predarae Nave.

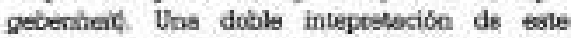
enncepto permita a Husecet examinar la histeria ccmo posbilisad y la historia somo efectivided. Lo cual agnifica qui, a trowis de las multiples tealluedes que la hiscorie nos pone de manifiesto paean condidanea do pocthilidad para qoo podamoe tener un heriacnte ristldice. Adambe, en telacica tanto on la pociblicind como con ha efuctividad, ts potibie trazar dos distircicnes ulesiones. Asi, ase pueden distinguir castro nivalea difarentes de la interpantacibe da la bistnria: If el nivel de las cordictones de poeinadad, esto 68 , de la implicactón de los otros, el tiempo y al munde, \&) el mivel de les condicicoes perviae pura la edoctivasucian, esto ea, de ta potoconeratiritad, is tradicioselisacian $y$ la tlecra; 3) el nivel de la eloctividad de a historia, esto es, de uns focma de la generatididat, una tradicke y us tariterios, 7 4) el thvel do la genera-

; Thivereddad de Buenes Aires.

1 Ia sigla correspoode, con indieación de volumen y págtna, a Bdmund Hussorl, Ossammalte Werke - Husestiana, wols. I-XXX. Decdrects, / Boseon / Loedon, Kluwer Academic Publisbars Icon antenoridad: Den Haas, Martinus Nijboin, 1960-1996. Los mazuaczive son chados de acuerdo con la

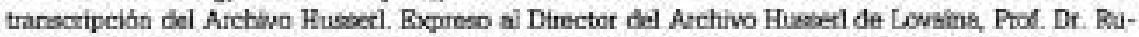

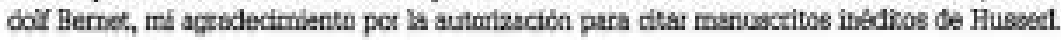

\begin{tabular}{|l|l|l|l|l|l|}
\hline VRRITAS & Pocto Alegre & v. 45 & n. 1 & Março 2000 & p. $13-35$ \\
\hline
\end{tabular}


husserliana segue este escalonamento e clareia cada nivel segundo três dimensões. De acordo com este esquema tentaremos, nas considerações a seguir, desenvolver a explicitação husserliana do horizonte histórico. tividad espiritual racional, de "la" tradición y de la representación de "un" mundo. El camino de la investigación husserliana sigue este escalonamiento y aclara cada nivel según tres dimensiones. De acuerdo con este esquema intentaremos en las siguientes consideraciones desarrollar la explicitación husserliana del horizonte histórico.

\section{Modos del predarse}

1.1. En primer lugar, ocupa un lugar muy significativo la diferencia entre dos modos del predarse en el Manuscrito A VII 11 (1932). La "estructura del predarse en el primer sentido" abarca un estilo familiar de constitución del mundo con una determinación solamente limitada del proceso histórico en relación con el desarrollo de la vida individual desde la infancia y el desarrollo elemental de la vida intersubjetiva. Remite a elementos en la estructura de mi vida, de los otros y del mundo, mientras que el predarse en el segundo sentido remite a adquisiciones en el desarrollo de estos elementos. Husserl subraya que la palabra "posibilidad" es ambigua, ya que puede ser "un título para un espacio de juego disyuntivo predelineado" o "un título para una multiplicidad abierta de posibilidades" (Ms A VII 11, 4b). Al segundo título se une el sentido secundario de la indeterminación como un tipo de permanecer abierto o de ser no decidido, que es determinable según "modos y niveles de la historicidad (Weisen und Stufen der Historizität)" (Ms B I, 16, Tr. 12). Aquí Husserl pone en claro que la estructura del predarse en el primer sentido solo es pensable como una forma estructural abstracta. En este sentido Husserl habla de "protohistoricidad" (Urhistorizität) (Ms A VII 11, 3b) y plantea el problema de una estructura predada del desarrollo:

"Tengo el mundo con un horizonte abierto, pero como el mundo que es para mí a partir de mi vida. A partir de mi vida tiene él (en conexión con los otros), tiene el mundo una estructura del predarse, y él fue en mi infancia uno distinto de ahora y se transforma constantemente como una determinada estructura del predarse. [...] A esto es inherente que en la conexión de la experiencia progresiva de cada uno y de cada humanidad comunalizada se produce por medio de un tradicionar (Tradieren) ante todo unilateral y luego recíproco un predarse progresivamente unitario, más rico, más ampliado [...]" (ib. 3ab).

Husserl expresa este enriquecimiento con el concepto de "segunda horizonticidad" (ib. 4b), que no delimita un espacio de juego disyuntivo predelineado. No sabemos nada de antemano respecto de este segundo modo de predarse. Así, Husserl se refiere a un concepto más estrecho y más amplio del predarse, y añade que la estructura del predarse en el primer sentido tiene un "ulterior horizonte transformado que solo se muestra en el desarrollo histórico de hecho" (ib. 4a). El segundo nivel del predarse da cuenta de las repetidas alteraciones que no pueden ser construidas de antemano. En este caso el mundo no solo es relativo al sujeto 
singular que lo experiencia sino también relativo a un entramado diferenciado de sujetos. Se debe señalar aqui que no sólo entran en cuenta los posibles caminos del desarrollo y la transmisión, sino también todos los desarrollos efectivos.

1.2. Este distingo entre un concopto estrecho y un concapta amplio de predarse se debe poner en peralelo con una diferenclación expuesta en la Vla. Moditación cartesiana. Fink contrapone un predarse que no proviene de la experiencia a un predarse que es referible retrospectivamente a una instución originaria. Un puro horzonte del predarse debe separarse de la cognoscibilicad acarca del mundo sedimentado en habitualidades. Esto significa gue un eselo ya acabado y pertocto antecede a toda experiencta. Puesto que todo devenir en el mundo aconteoe en coincldencia con este estilo y la experiencla del mundo se deserwuelve conrelativamente en un estilo aperceptivo que conesponde a este estilo de set, Fink puede afirmar: "El predarse del mundo cono perfectivided del estilo del mundo es un findice de una determinada historicidad, (...)" (HuaDoik II 2, 97). ${ }^{2} \mathrm{Asi}$, disponemos de un conocimiento previo del eatilo de ser del mundo, que se muestra seguin los dos lados do los puros horizontes de posiblidades y del predarse habitual del mundo $y$ que se ha do analizer seguin tree modos de preoogncecibilidad: el predarse de loe objetos para el sujeto humano, el predarse del sujeco humano para st mismo y el predarse de los horizontes temporales.

Hay una comprension previa de la articulación fundemental y tipice no salo de todo el mundo objetivo sino también de los actos, habitualidades, estados de ánimo, etc. Pero este segundo modo de predarse no se limita a los conocimientos inmediatos, alcarzables por medio de la relexión. Abarca también "todos los modos de transmision de experiencias, en los cuales nos apoderamos de un saber expenencial sobre nosotros [... $]^{*}$ (b. 207]. Tenemos una comprensicin preria en el modo del harizonte de las dimensiones del conocimiento mediato alcanzable por medio de los otros: "Antes de toda efectivización y plenfficación f́cctica son ya predades las dimensiones a partir de las cuales una experiencia excraña puede ser tranemitida y en las cuales yo puedo enviar mis vigencias de ser mediatas, en un estilo general de conocimiento del mundo (b. 99 ). Estas dimensiones de la transmistón de la experiencta extrahla stgnifican dos ocsas. Por un bdo, se da un conocimiento previo de los posibles caminos o vias de transmisión, esto es, de las formas de acceso por medio de las cuales recibimcs esta experiencia surgida de los otros. Bstos camincs de la transmisión corresponden a los otros presentes, los otnos ausentes en el horizonte de la simultaneldad y los otros en el horizonte de la sucesion. Por ctro lado, se da el prederse de la experiencia mecilata en cuanto tal, esto es, de aquello que recibirnos por estas vias. Si bay un predarse en relación con la ùltima via mencionada, el predarse no se limita al mundo presente. Asf, el mundo nos es prededo en la medida en que la medieckin de los otros bo extiende más alla del presente, y, en consecuencla, abarca la exdstencia del mundo antes de mi nacimiento y después de mi muerte. Con otras pelabras. el predarge se refiere no solo al 'qué' de to transmitido sino tam-

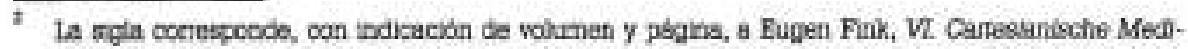
totion, Husostituna Dohumante II, Docdrocht / Bocton / London, Kluwer Academie Puklikhers, 1908. 
bién al 'cómo' de la transmisión. No se trata de un conocimiento determinado, fácticamente transmitido, sino de un conocimiento de los marcos predados para todos los conocimientos transmitidos. Esto significa que "el contexto total estructural de la existencia humana" nos es previamente dado: "El sujeto es predado a sí mismo como hombre en el mundo, entre el nacimiento y la muerte, existiendo en comunidad con los otros, en medio de una naturaleza sobrepoderosa, etc." (ib. 208). Que la comprensión del mundo predado encierre una comprensión del ser del mundo antes de mi nacimiento y después de mi muerte constituye para Fink la razón por la cual las experiencias histónicas presuponen "el horizonte del predarse de la "historicidad" (ib. 101).

El predarse de la experiencia externa y el predarse de la autoexperiencia constituyen el predarse del mundo. La vinculación de ambos predarse reside en el "predarse de los horizontes temporales". Según Fink, nos movemos siempre ya en una precomprensión de la estructura formal y la articulación del tiempo. La estructura formal concieme a las relaciones temporales fundamentales de presente, pasado y futuro y a sus referencias reciprocas. La articulación concierne al pasado del mundo antes y después de mi nacimiento y del futuro del mundo antes y después de mi muerte. Por tanto, se encuentra predado también el estar encajado, articulado, ordenado (Eingebettetheit, Eingliederung, Einordnung) de mi tiempo de vida subjetivo en el tiempo del mundo. Puesto que el tiempo del mundo engloba la inmanencia y la trascendencia, toda consideración de la temporalidad de los actos subjetivos es solo una abstracción. En una nota marginal a esta afirmación de Fink, Husserl añade: "Cada uno tiene el tiempo del mundo y en él temporalmente ordenado (eingeordnet) su tiempo inmanente. Pero mi tiempo inmanente abarca intencionalmente a los otros y a sus inmanencias y así accedemos ya al camino hacia la intersubjetividad en la pregunta por el predarse" (ib. 212 n.). Aquí están implicadas dos cosas. Con la indicación de un englobamiento de los otros está en juego una experiencia subjetiva del tiempo. Con la referencia a un ordenamiento del tiempo inmanente por el contrario aparece en primer plano el tiempo común de la historia. Por un lado, el tiempo inmanente abarca todos los tiempos; por el otro, es abarcado por estos. En este sentido se expresa Merleau-Ponty aun con más énfasis cuando, en relación con la noción husserliana de horizonte, afirma que "aquel ante el cual se abre el horizonte, está incluido y encerrado en él". ${ }^{3}$ Esta cita subraya no solo que el tiempo inmanente está subordinado al tiempo de la historia sino también que nos proporciona el acceso al tiempo del mundo.

Ahora quisiera intentar aclarar lo que significa la unidad universal de posibilidades mantenida constantemente en la conciencia de horizonte. Pues el predarse del mundo es ante todo una comprensión de posibilidades que presupone la conciencia de horizonte: "Toda forma de la conciencia de horizonte es una forma fundamental de la intencionalidad. Solo a partir de la esencia de la intencionalidad en cuanto tal, interrogada y aclarada filosóficamente, puede ser comprendida la conciencia de horizonte del predarse" (HuaDok II, 2, 205). 


\section{La posibilidad de la historia}

Ahora debemos examinar la estructura más general e indeterminada que, según Husserl, exhibe el horizonte histórico. Es inherente al sentido más originario del predarse que abarque tres particulares estructuras de horizonte de la intencionalidad. Ellas se presentan como la más pobre y limitada determinación para la historia.

2.1. Ante todo se debe mencionar la implicación del horizonte temporal. Husserl la conecta con la sedimentación de la sintesis retencional que es inherente a la génesis intencional. Y teniendo en cuenta el "incesante devenir" de la conciencia y la historia como un escalonado "constituir [...] de formaciones de sentido siempre más elevadas", afirma: "La historia en su sentido habitual en su relación con la cultura humana es solo un nivel más elevado [...]" (Hua XI, 218-219).

2.2. En segundo lugar debemos prestar atención a la implicación de los otros y su relación con el tiempo subjetivo. Así como una doble intencionalidad de la retención juega un papel en la constitución del flujo de la conciencia (cf. Hua X, 80 ss.), se podría asignar también a una doble intencionalidad de la apresentación un papel en la constitución de un flujo intersubjetivo. La secuencia impleción intuitiva - intención vacía - protoforma de la intención puede ser mostrada en cada curso individual de tal manera que se manifiestan los siguientes fenómenos: 1) la rememoración como presentificación de los propios actos pasados y como impleción de la retención; 2) la retención como una protoaprehensión que se dirige a las fases temporales de los actos pasados por medio de la intencionalidad transversal; y 3) la retención como una protoaprehensión que se dirige a las correspondientes fases del flujo absoluto por medio de la intencionalidad longitudinal. Junto a esta estructura subjetiva se puede delinear una ulterior estructura que muestra cómo el camino de la apresentación corre paralelo al de la retención. Es fácil indicar los dos primeros momentos de una secuencia paralela: 1) la impatía auténtica como presentificación de los actos de otro yo e impleción de la apresentación (cf. Hua XIII, 455 ss., 478 s.); y 2) la impatía inauténtica como la apresentación vacía del yo extraño en conexión con el cuerpo propio percibido. Surge aquí el problema acerca de si y cómo se da también un tercer miembro en esta secuencia.

En analogía con la retención de las fases del flujo absoluto se ha de esbozar la idea de una apresentación que no se dirige hacia la otra subjetividad mediatamente por medio de la presentación del cuerpo extraño. Una apresentación más originaria da cuenta de la unidad de los diferentes cursos, así como la intencionalidad longitudinal de la retención o del flujo absoluto da cuenta de la unidad del flujo subjetivo. Mientras que su orientación transversal se dirige a la subjetividad efectiva a través de la presentación del cuerpo propio extraño, su orientación longitudinal se vuelve mediatamente hacia las posibles otras subjetividades por medio de la ficción como diferente del posible poder ser otro del yo (cf. Hua XIV, 154 s.). Este modo de apresentación no se apoya sobre la aprehensión analógica del cuerpo propio extraño sino sobre una red de implicaciones, esto es, sobre la coincidencia de toda posibilidad de otro yo con la posibilidad de mi ser otro: "[...] así 
obtenemos posiblidades para cuerpos propios extranics no percibidos, pero eventualmente percepsibles en el desarrollo ulterior, $y$ asi un horizonte de otros, no a partir de la impata efectiva sino a partir de le impatia que se encuentra dontro de nuestras capacidades" (Ms C 17 V, 76ab). Huaserl habla de una "protoimpatia" (ib., 84b), de "ia originariedad de la impatia" (Hua XV, xlix), en conexción con un tipo de apresentación o, por decitlo asi, de "apresentación longitudinal": "Porto a todos los otros en mi como apresentados on si mismoe $y$ a set apresentados $y$ como sustentando en ai del mismo modo a mi migmo. [..] asi como yo y luego

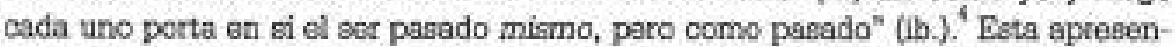
tación "longftudinal" de la subjetividad extraña, no considerada explicitamernte por Husserl, inicia una amplia revisión del cantramiento en lo propio, y, en tanto motivo contrario, se acrecienta en los sigujentes niveles del analisis - que consideraremos luego en detalle - por medio de una intencionalidad impulaiva universal y las diferentes formas de las generatividades de nivel superior."

2.3. En tercer lugar se presenta la implicación del mundo en cada experiencia. Husserl procura explicitar un uno-con-otro y un uno-en-otro de horizontes y una temision de los horizontes en sf primeros a los ulteriores, de modo que el mundo se abre como horizonte de todos los horizontes a partir de cada cosa real experienciada y su propla horizonticidad. EI desvelamiento de las horizontes coincide con el desvelamiento del mundo, que se tranforma constantemente y a la vez permanece unitanamente de un modo no temático en la experiencia de lo singular. Pero esta mera conciencia de horizonte del mundo, que esta implicada siempre en nuestra experiencia, puede ser explicitada por medio de una representación del mundo (Weitworstellung) y eventualmente aclarada por medio de intulciones La tematización es una excepción, porque aquello con lo que contamcs la mayor parte de las veces es solo un horizonte total latente. Como excodencia, el hodizonte del mundo va más alla de todas las representaciones.

\section{Las condiciones previas de la efectivización}

Ea relación con una estructura más determinada cet peimer sentido del peedarse, y en comelacion con las tres dimensiones de a implicación intencional del tiempo, los otros $y$ el mundo, Hussen pone de relieve tres dimensiones particular-

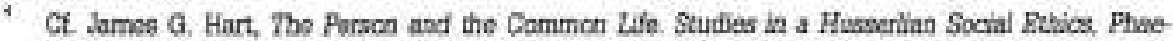

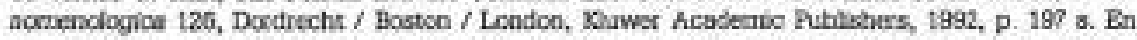

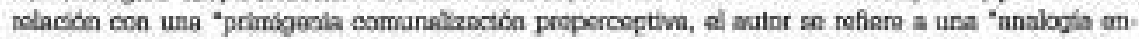

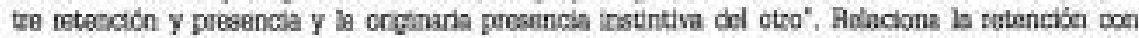
"una pecoconciencha de comuridad y an diferentes pocutaridsdne y relacikos foin Vor Bewusstsain

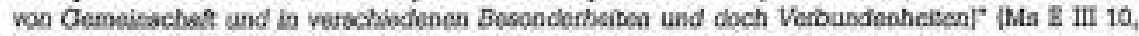

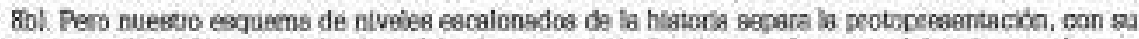
tnteccienalidad begitudinal sobee el fundaments de la fockon trangormante dei poder ser de otra

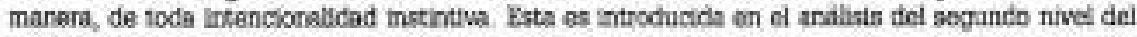
predurse.

1 Para una indicacion sobee esta cuestion, wease Bernhard Waldenfels, Topographis des Fromdan Station zur Fnancmenologle des Proxiden 1, Brankfurt a. M, Suhrkamp, 1997, p. 90. 
mente importantes: la protogeneratividad, la tradicionalización y la tierra. Ambos niveles del análisis de la historia se encuientran comprendidos en el único concepto de condiciones de posibilidad, pero el segundo nivel se encuentra predado de una forma y con una competencia distintas que las de la mera implicación, porque tiene que ver con las condiciones previas para la efectivización de la historia. A partir de aquí el análisis debe comenzar con la dimensión de los otros porque el tiempo queda sujeto a los vínculos intersubjetivos.

3.1. A la mera implicación de los otros corresponde en este nivel la protogeneratividad: "El mundo predado en su omnitemporalidad abierta infinita me contiene en el encadenamiento de las generaciones [...]" (Hua XV, 583). Husserl observa que cada hombre es "para sí persona en el nexo generativo infinitamente abierto, en el encadenamiento y ramificación de las generaciones" (ib., 178). Puesto que tiene un pasado y un futuro de generaciones, y se conoce como el miembro ahora viviente de la cadena de predecesores y sucesores, el hombre vive en el "horizonte de su generatividad" (Ms K III 3, 38a). ${ }^{6}$ Aquí se plantean tres problemas que son particularmente importantes para la consideración del fenómeno de la historia. En primer lugar, emerge el problema del ser antes de la generación, esto es, la pregunta retrospectiva por el pasado del mundo antes de mi nacimiento. Según Husserl, me represento el pasado "sobre el fundamento de la primera inmediata y la relativamente inmediata intersubjetividad" (Ms C $17 \mathrm{~V}, 91 \mathrm{a})$, por medio de una ampliación, a fin de esbozar el desarrollo de los organismos hasta el organismo humano. Es posible comprender el desarrollo ontogenético y filogenético por medio de "transformaciones de los modos de representación" (ib.), esto es, por desconstrucción y modificación. Esto significa que nos imaginamos los modos en los cuales los animales se relacionan con el mundo en el modo de su representación animal. En el límite podemos avanzar hacia la vida de las plantas como modificación en el modo de una vida sin cinestesias.

En segundo lugar, se debe dar cuenta de la "forma de la generatividad" (Ms K III 18, 18b) en relación con la ramificación. Hay marcos para el encadenamiento de las generaciones, y así llegamos al "problema de las generatividades separadas" (Hua XXIX, 62). Husserl se refiere a las diferencias entre una familia y otras familias, entre una comunidad de familias como comunidad de una aldea o una tribu y otras comunidades, entre una asociación de aldeas como nación y otras naciones, entre una comunidad de naciones somo supranación y otras supranaciones. En estas diferentes direcciones se despliega la separación de la generatividad.

Por último, aparece claramente en el centro de la investigación la consideración de niveles de la generatividad. La diferencia entre una generatividad inferior y superior se conecta con el contraste entre sociedades que están construidas sobre

6 Un texto de la Crisis expresa claramente este punto de vista: "[...] esta forma de la generatividad y la historicidad es inquebrantable así como la forma inherente a mí como yo singular de mi presente perceptivo original como presente de un pasado rememorativo y un futuro previsible" (Hua VI, 256). Sobre el concepto de generatividad, véase Anthony J. Steinbock, Home and Beyond. Generative Phenomenology after Husserl, Evanston, 11., Northwestern University Press, 1995, pp. 170-270. 
un "Impulso hacta la solictud por las nitios, impulso famitrar o impulso de comunidad" (Ms K MI 7, Tr. 10, y sociedades que se configuran sobre la base de un acuerdo en torno de una meta coman (ct. Hua XIII, 107 s.; XV, 67). El universo de los instintos se identifica con una intenclonalidad universal cuyo despliegue esté ortentado por habitualidades originarias que antecesen a jas que provienen de la sedimentación de los actos. Al respecto Husserl habia de una preadquisicion y preconstitución y se pregunta si "la humanidad total debe ser una unica generatividad, la unidad de una historicidad en el mas amplio sentido, aun cuando 10 enlazante esta oculto" (Hua XXIX, 62 s.). Esta es la base para genetativicades de otden superior, que se presentan en cada caso en un enlace con el nexo protogenerativo:

"En su protoinstinto porta en st cada aufato singular todo este desarrollo no ccmo su desamollo solijgista sino como desarrollo de la humazidad - cono desarnollo de la omnicomunidad trascandental, la de los sujetos trascondentales -, esto es, porta en si "implictarnente" todos bes otros qve pueden saitive al encuentro, y todas sus operaciones, todo el mundo como hemanizado, como mindo culturar (Mis A VL, 34, 37a).

3.2. Otra dimensión concierne al fenćmeno de la tradicionalidad. Cadia hombre tiene su historicidad individual, que se escinde en una historicidad de la inlancia temprana en el cuespo materno y posteriot al nacimiento, y en una historicidad infanti posterior que se extiende hasta la madurez. Este desarrollo se ajusta ya a la forma protoinstitución-sedimentación-Jeirstitución y se ordena segủn la unidad de la conexión entre la madre y al hijo, los niños unos con otros, los mayotes y los niños dentro de la "historicidad de una familia" (cf. Ms D 14, Tr. 58), y finalmente en la unidad de la conexión de los mayores unos $\operatorname{con}$ otros en la unidad de un grupo humano como "familla en sentido amplio" (Ms A V 24, Tr. 31). Puesto que es "una constante unidad de 'autoconservación'", la comunidad goe ha legado a ser generativamente tiene la correspondiente "unichd de una 'historia', de una tradiçón comunitaria* ib . de manera que al mundo circundante personal le cotrespcnde "una historicidad abstracta" (Hua XV, 13:n). Con el uno-con-otro de los adultcos ingresa cada hombre claramente en una historicidad comunitaria y recibe la herencia, que reside en ella, de generación en generactón. Bl nivel de madurez. alcanzado para la historicidad índividual es el comienzo de la auténtica vida en la omnisocialidad e historia de los hombres.

A esta protohistoria pertenece tambjén la comprensión de la finalidad. El niño vive en un mundo familiar y se encuentra rodeado por otros que actían en función de metas y objetos gue tienen el carácter de metas. For eso aprende a comprender los objetos de uso taleológicamente como objetos que se asocian con una meta: "La pregunta por el por qué es la pregunta originaria por la "histcria"' (Hua XV. 420). Asi se desarrolla una protencionalidad que tiene en cada caso una meta cercana y una progresiva satisfacción "sin que una determinada meta final fuera explicitamente consciente junto a la meta en cada caso próxima" (Ms K III 3, 50a). Esta protencionalidad está alajada de la historia en sentido estricto, porque cesta presupone una más amplia comprensibilidad intersubjetiva en torno de fornacio- 
nes asociadas con metas. Sobre esta historicidad originaria se edifican otres formas que despliegan la forma de la protencionalidad por mectio de formaciones superiores que implican fines en sentido estricto:

"A esta protoforma de la histcricidad (Uurform der Coschichtlichkeit) la Ilamamos bistaricidad etiginaria, pootencional (ursprüngliche, protentionale Geschichtlichkeit). Ella es tambien en un nivel superior base para cada historicsdad superior (Unterlage fiur jede hohone Geschichtaichkeit), Y cada histocicidad superior adcpta siempre en cicrto modo la forma de la "peotencionalidad", excedida entcrces de nuevo por posibles confyutaciones tuevas superiores" (Ms K III 3, 50a).

Aqul podemos destacar una convergencia con el pensamiento de Heidegger. Bajo el titulo "La constitucion fundamental do la historicidad" se considera en Ser y tempo un acontecer (Geschohon) "que deternina la existencia como histórica". Este acontecer ahlade a la temporalidad loe rasgos de la "prolongación (Erstreckung)", la "movilidad (Bowogthoit)" $y$ la "perduractón (Behanrlichkeit)" que aclaran el "nexo de vida (Leborazusammonhang)" del Dasein. Ast, la historicidad está fundsda sobre una estructura de acontecer, que, por su parte, está arraigada en la temporalidad, esto es, tiene sus condiciones tamporales de poeibliidad. En la descripción husportiana de las condiciones previas para la posibalidad de la histcria aparecen tres rasgce fundamentales análogos tambien en relación con el "nexo de vida" (Hua VL, 152). El procaso de la maduración, la fluente "mcvilidad de vigencias cooporantes (Bowsglichkoit mitfungiorender Goltungen)" (b.) y la reactivación de sedimentaciones configuran los paralelos de la prolongeción, la movilided y la perduración.

Sc debe observar también aquí que la tradicionalidad coincide con el primero de los tres conceptos que Ficoeur reúne bajo el titulo de tradicion. Junto al concepto material de las diferentes tradiciones y el concepto apologétio de "la" tradición particularmente valorada, Ricoeur se tefiere, apoyándose en $\mathrm{H},-\mathrm{G}$. Gadamer, a la tradicionalidad para designar el estilo formal del encadenamiento en la relación con el pasado. Se trata de una reciprocidad y tensión entre la eficacia del pesado y el ser afectado, esto es, de la dieléctica del alejamiento y el acercamiento, de la distancia y la mediación, que se contrapone igisalmenta a la eliminactón de toda distancis o a un espacio intermedio insalvable."

3.3. La tercera dimensicn en este nivel concierne a la tierra como suelo para la historicidad indivichal y la histocicidad comuniteria dentro del horizonte de la generatividad. De este modo el mundo como horizonte universal reclbe una clecta determinación por medio de la "forma de aperición cialo-tierra" (Ms A VII 1, 23b), que permanece constante, y se muestra, como dice Hussenl, como "horizonte

3. Martan Heidogger, Sein and Zert, Tubingen, Mex Niemeyer, "1959, p. 382. So erweist scta im

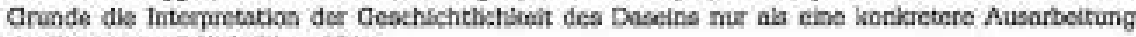
der Zeatichikeit" ib.L. Ct. p. 3748 .

,

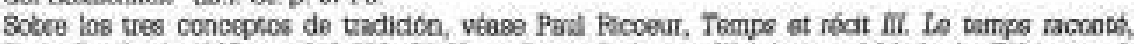

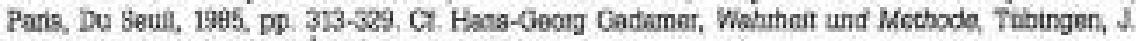
C. B. Motr IPaut Stabecil, ${ }^{3} 1972$, p9. $275-290$. 
un "impulso hacka la solicitud por los ninos, impulso familiar o impulso de comurudad" (Ms K III 7, Tr. 10), 7 sociedades que se conflguran sobre la base de un acuerdo en tomo de una meta comun (cf. Hua XIII, 107 s.; XV, 57). El universo de los instintos se identifica con una intencionalidad universal cuyo despliegue está orientado por habitualidades originarias que antaceden a las que provienen de la sedimentación de los actos. Al respecto Husseri habla de una preadquisición y preconstitución y se pregunta si "le humanidad total debe ser una única generativichad, la uridad de una historicidad en el más amplio sentido, aun cuando lo enlazante esta oculto" (Hua XXIX, 62 s.). Esta es la base para generatividades de arden superior, que se presentan en cada caso en un enlace con el nexo protogenerativo:

"Bn su protoingtinto porta en al ceda aujeto singuler todo eete deeerrolo no como

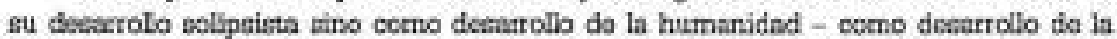
omnicomunidad trasoendental, la de los st,etos trascenbertales -, esto es, porta en si "implictamente" todos los otros qua pueden salirle al ancusntro, y todas sus operacioDes, todo el mundo como humanizado, como mundo cultutal" (Ms A VI, 34, 37a).

3.2. Otra dimensión concierne al fenómeno de la tradicionalidad. Cada hombre tiene su historicidad individual, que se escinde en una historicidad de la infancia temprana en el cuerpo matemo y posterior al nacimuento, y en une historicidad infantil posterior que se extiende hasta la madurez. Este desarrollo se ajusta ya a la forma protoinstitución-sedimentación-reinstitución y se ordena según la unidad de la conexión entre la madre y el hijo, los nifios unos con otros, los mayores y los niños dentro de la "hissoricidad de una famlia" (cl. Ms D 14, Tr. 58), y linalmente en la unidad de la conexión de los mayores unos con otros en la unidad de un grupo humano como "familia en sentsdo amplio" (Mss A V 24, Tr. 31\}. Puesto que es "una constante unidad de "autoconservacion'", la comunidad que ha llegado a ser generattivamente tiene la correspondiente "unidad de una "historia', de una tradición comunitarla" (ib.), de manera que al mundo circundante personal le corresponde "una historicidad abstracta" (Hua XV, 138n). Con el uno-con-otro de los adultos ingresa cada hombre claramente en una historicidad comunitaria y recibe la herencia, que reside en elta, de generacion en generacion. B nivel de madurez alcanzado para la histcricidad individual es el comienzo de la autentica vida en la omnisocialidad e historia de los hombres.

A esta protohistoria pertenece también la comprensión de la finalidad. Bl niño vive en un mundo familiar y se encuentra rodeado por otros que actúan en función de metas y objetos que tienen el carácter de metas. Por eso aprende a comprender los objetos de uso teleológicamente como objetos que se asoctan con una meta: "La pregunta por el por qué es la pregunta originaria por la 'historia'* (Hiua XV, 420). As so desarrolla una protencionalidad que tiene en cada caso una meta cercana y una progresiva satisfacción "sin que una determinada meta final fuera explicitamente consciente funto a la meta en cada caso proxima* (Ms K III 3, 50a). Bsta protencicnalidad está alejada de la histcria en sentido estricto, porque esta presupone una más amplia comprensibilidad intersubjetiva en torno de formacio- 
nes asociadas con metas. Sobte esta historicidad originaria se edifican otras formas que despliegan la forma de la protencionalidad por medio da formaciones superiores que implican fines en sentido estricto:

"A esta protoforma de la historicidad (U)form der. Ceschuchtilichkeit) la limamos

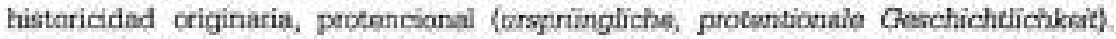
Blla es tambien en un nivel superior base para cada histocicidad suparior (Unterlage für jede hchere Gaschichtlichikeitf. y cada historicidad superior adopta siempre en cierto modo la forme de la "protencionalidad", exoedidia entonces de nuevo poc posibles configuraciones nucvas superiares" (Ms K III 3, 50a).

Aquif podemos destacar una convergencia con el pensamiento de Heidegger. Bajo el titulo "La constitución fundamental de la historicidad" se considera en Sor y tempo un aconteoer (Geechehen) "que determins la existencla como histónica", Este acontecer añade a la temporalidad Jos rasgos de la "prolongación (Erstreckung)", Is "movilidad (Bewegtheit)" $\mathrm{y}$ la "perduración (Beharzlichkeit)" que aclaran el "nexo de vida (Lebonszusammenhang)" del Dasein. Asf, la historicidad esta fundada sobre una estructura de acontecer, que, por su parte, está arraigada en la temporalidad, esto es, tiene sus condiciones tempcralas de poeibllidad. En la descripción hussertiana do las condicionos peevias pera la posibilicad do la histocia apareoen tres rasgoe fundamontales análogos tambión on relaciót con el "nexo de vida" (Hua VI, 152). Bl procseso de le maduración, la fluente "movilidad de vigencias coopecantes (Bewogichkeit mitfungiorander Goltungan) (ib.) y la reactivación de sedimentaciones configuran los paralelos de la prolongación, la movilidad y la perduración.

Se debe cbservar tambión aqui que la tredicionalidad coincide con el primero de los tres concoptos que ficceur reúne bajo el titulo de tradición. Junto al concepto material de las diferentes tradiciones y el concepto apologettico de "la" tra. dición particularmente vulorada, Ricoeur se refiere, apoyándose on H.-O. Gadamer, a la tradicionalidad para designar el estilo formal del encadenamiento en la relación con el pasado. Se trata de une reciprocidad y tensión entre la eficacia del pasado y el ser afectado, eato es, de la dieléctica del alejamiento y el acercamiento, de la distancia y la mediacsón, que se contrapone igualmente a la eliminación de toda distancia o a un espacio intermedio insalvable. ${ }^{B}$

3.3. La tercera dimensión en este nivel concjerne a la tierra como suelo para la historicidad individual y la histoticidad comunitaria dentro del horizonte de la generatlvidad. De este modo et mundo como horizonte universal recibe una cierta determinación por medio de la "forma de aparición cielo-tierra" (Ms A VII 1, 23b), que permanece constante, y se muestra, como dice Hussect, como "horizonte

Martin Hadogger, Stin und Zet, Tutingen, Max Niemeyer, "1963, p. 392. "So erweist stch im Grunde dis intergcetatioa der Deschichtichkeit des Daseine nu ab ese woniretere Aasastaitury der Zeittichker" (b) Cf p. $374 \mathrm{~g}$.

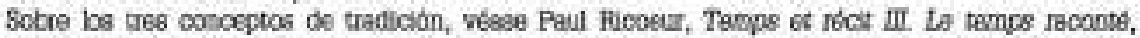

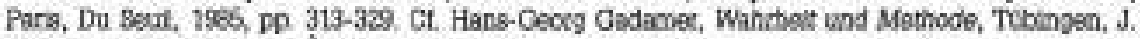

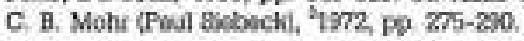


terrestre" (Hua XV, 206) y "suelo vital de la humanidad" OMs A VI 10, 26a). Aun cuando los diferentes pueblos están separados, cada pueblo tiene como patria la misma tierra. Cada comunidad cultural unitarta es una "unidad cultural localizada*, porque esta reterida a la unidad de un territorio, y, puesto que la tierra conflgura un mismo mundo para todos, Husserl puede escribir: "[...] todas los desarroJos, todas jas historias relativas tienen por tanto una unica protohistorla (Uthisto[5e), cuyos episodios son".

Con sus consideraciones relativas a las condiciones previas de la efectividad de la tistorla, Hussen anticipa el "primer movimiento de la vida humana" de Jan Patocka en sus rasgos fundamentales. Po medio de este movimiento de anciaje se ectan ralces en el mundo porque los ocros nos acogen y protegen, se ocupan de nuestras necesidades y configuran una patria originaria de manera que "la tierra sea cálida, cordial y amistosa". Poc otro lado, en el movimento secundario de la inserción como en el predarse en el segundo sentido, los otros se encuentran presentes "en sus obras, en los sedimentos de su actividad, en las situaciones que ellos han creados o cuyos componentes ellos forman con nosotros". Es evidente que esta concepción debe mucho a Husserl, pero hay un desplazamiento del énfaais porque Patocka pona en primer plano el cuidado de los niños y excluye la consideración del enlace entre protogeneratividad y generatividad.

Se debe señalar también que este tema fue un importante punto de partida para el énfasis de Merleau-Ponty acbre "la preexistencia del ser natural siempre ya ahf." "Esta facticidad de la naturaleza significa cue la profundidad de cada presente encierra algo que se encuentra más alla del alcance de nuestras rememcraciones, $y_{1}$ por tanto, solo puede set caracterizado indirectamente como pesedo de todoe los pasadoe. Por eso h estnuctura tierra-protogeneratividad tostimonia on Morieau-Ponty una "Uthistorie corporal" y significa "la simultanes Unstiftung de tlempo y erpacio que hace que haya un paieaje historico y una inscripción cuast geogrífica de la historia". A ello affade Merleau-Ponty: "Probloma fundamental: la sedimentación y la reactivación", ${ }^{12}$ Pero esto ya nos conduce a una nueva dimansión del predarse.

\footnotetext{
Edmund Hussed, "Grundegende Untersuchungen sum phancrsenolognachen Urspoung der Raam-

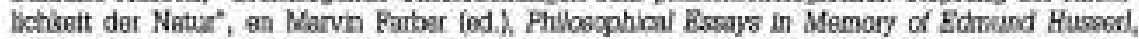
Ocesowood Prese, New Yoxk, 1968 (Reimp.: Cambrisge. Msss, Harvwd Uniseraky Prese, 1940, D. 319.

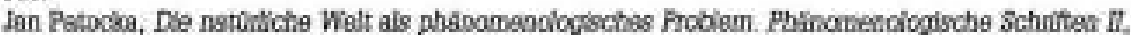
trad de B. y R. Melvilis, Suttyart, Kontt-Cotts, 1991, p. 139 C. pD. 213 as.

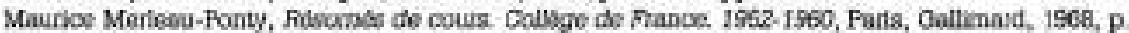

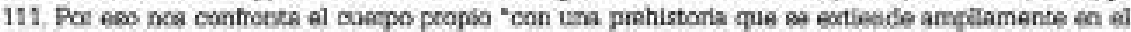
acontecer de la satumalecea (Bamhard Waldentela, Grenzen der Normalksinang Studien zur

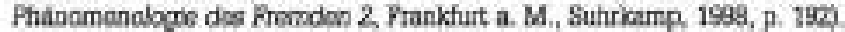

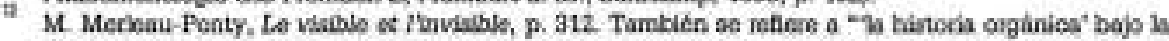

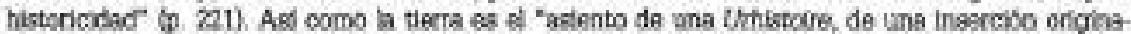
ra", la naturalaza es "esta oportumidad cfrecida a la corporalidad y a la intergublettvidad" Maunce

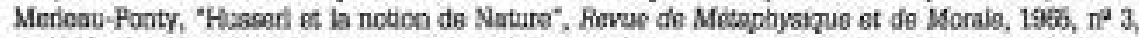

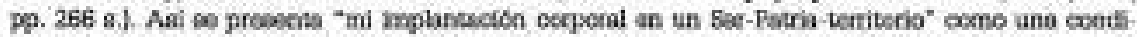
dín previa para la electividad de li hüatoria: "Cue h histodia tenge un sentido, que sea unica, que
} 
Las tres condiciones previas para la efectivización de la historia complementan el nivel de la mera implicación. Frente a la indeterminación de la implicación de las oparaciones pasadas, los otros y el mundo, la tradicionalización, la protogeneratividad y la tierra introducen componentes de deteminacion. Respecto de los dos niveles de este predarse en el primer sentido se puede recordar que Merleau-Ponty ha caracterizado el ser vertical o salvaje como "este medio pre-espiritual sin el cual nada es pensable, n siqujera el espiritu, y por el cual pasamos los unos en los otros, y nosotros en nosctros mismos para tener nuestro tiempo". "Protogeneratividad, tradicionalidad y tierra reptesentan una indeterminación de la posesión, 0 , mejor dicho, sehalan el punto cero en la gradactón de la cognoscibilidad respecto de las adquisiciones de la historia. Son dimensicoes que deben ser plenificadas por las generatividades de orden supenor, las tradiciones y los territorios. Pero con ello pesamos dol predarse en el ptimer sentido al predarse en el segundo sentido. Ea signifcado de este pasaje se nos aclara al plantear la pregunta por lo que diferencia la historia posible de ha historia efectiva. En la mectida en que el predarse en el primer sentido se caracteriza por les meras postbilidades, y permenece por elb indeterminado, no puede asociarse con ninguna posestón de la histcria efectiva. Ahcra considerasemos aquello qua con las determinaciones de protogenerativiad, tradicionalizaçón y terra solo ha comenzado a ponerse en marcha. Elas contienen las condiciones previas de la efectividad de la historia, sin que esta sea darivable de ellas.

\section{La efectividad de la historia}

Husserl distingue la tradicionalidad o "tradicionalización" como una continuidad y unidad de pasados que se implican uno en otro hasta el presente, esto es, "la forma total "presente histórico en cuanto tal", tiempo histórico en cuanto tal", de las "configuraciones particulares de la cultura ordenadas en su sentido histórico unitario como tradición y transmitirse viviente* (Hua VI, 380). En este texto se refieja la diferencla entre predarse en el peimer sentido y predarse en el segundo seatido. Con el segundo, la tradicionalidad se desarrolla hasta convertirse en una tradición efectiva en conexión con una determinada forma y nivel de la generatividad. Con ello aparece "una primera historicidad", "dentro de la cual el estllo notmal en cacla caso fáctico de la existencia creadora de cultura en todo ascenso o hundimiento o estancamiento pemanece formalmente el mismo" (Hua V, 326). ${ }^{14}$ Bsto significa un enriquecimiecto del concepto de suslo. La tierra es lo que no

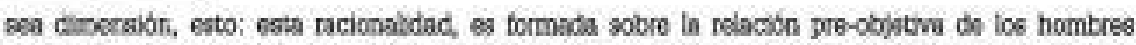

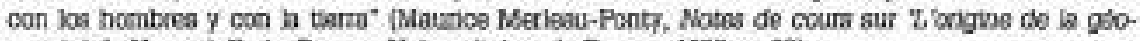

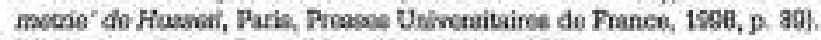

M. Vesleau-Ponty, Le vitble ex l'wrisicle, D. 257.

Bn su cara a lary-Brahl, Hussent distingue un "estancrmiecto creme de hatoda" de "una vida

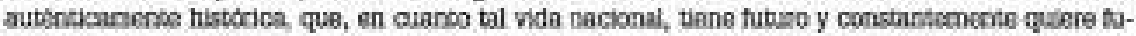

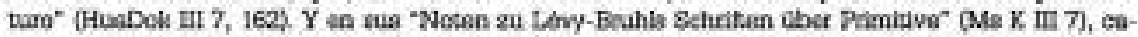
recteriza ls ausentica hisiona crmo "unidad de una humenided, que se 'desacrolla", esto es, de una hursaridad que tisne "un bscizonte de futuro y de voluntad pas su vida" 0 , Tr. 7 . 81 "estanca-

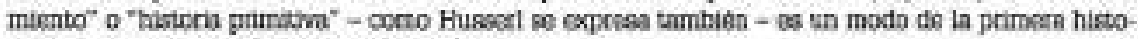
rididad, ca una cunstion que queda equi en maspenso. 
puede estar ausente como componente cel predarse en el primer sentido. Es el protosuelo (Uiboden) "sobre el cual se desarrclla la vida universal y al cual se incorpora de inmediato toda operación de la misma, toda actividad I...J" (Ms A VII 20, 27 g). Con esta actividad, el mundo se convierte en un suelo de sentido y validez, y esto significa que toda sociedad tiene "como suelo el mundo circundante generativo del caso con su nosotros generativamente viviente" (Hua XXXX, 62). Este suelo ulterior pertenece al nivel del predarse en el segundo sentido.

4.1. Sobre el fundamento de la generatividad orgánica se desarrolla una generatividad especificamente espiritual como "gencratividad de orden superior" o "cadena de generaciones de orden superioc" (Ms K III 4,32b) por medio de la institución de metas comunes perdurables. Asi se estahlece "un mundo de la vida de generaciones sucasivas con la unidad de una tradición histćcica [...]" (Hua XV, 205). Cada bombre se sabe "como miembro ahora viviente del encadenamiento de sus predecescres, su vide incluida en su enlace vital, como herencia de su cutura, y configurándola progresivamente" (Ms K III 3, 38a). Puesto que continuan viviendo como sdquisticiones sedimentadas, y ejercen una fuerza de motivación, las formaciones culturales enlezan la cadena de las generaciones por medio de un morimiento que va de la formeción de sentido a la reactivación por medio de la sedimentación de sentido. De esto se sigue que las intencicnalidades singulares, qua pasan a través de todos los presentes históricos, se enlazan en tazón de que están crientadas por una posesión, respecto de la cual no solo se puede preguntar retrospoctivamente por la intencionalidad que la instituyó orginariamente sino que se pueden explicitar los horizontes latentes.

En este nivel de la historicided comunitania "se repite el juego de desarrollo de la infancia y madurez como comienzo" (Ms A VII 11, 106). Pero Huseerl sulbraya: "La vida humana está explicitamente referida a su muerte, peco tambión referida a su historia humana, [...]" (Ms E III 10, 11b). Lo que importa acqui es que el cambio de los sujetos, en la medida en qe ingresan fuevos sujetos y orcs se apartan, no tiene que ver tanto con el nacimiento $y$ la muerte sino mís bsen, sin que entre ambos casos se pueda trazar un limite estricto, con la alteracion de los portadores maduros de valldez. Junto a esta "función de oo-validez de la cadena de generaciones" (cf. B III 11, Tr. B) se presenta como rasgo fundamental de la historia el entrelazamiento de muchas relaciones activas diferentes entre los hombres. En contraste con un acontecer intersubjetivo pasivo y solo inmediato, Husserl se refiere a "el acontecer producido

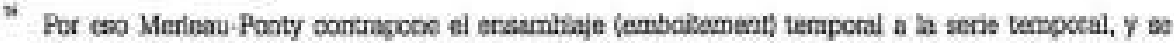

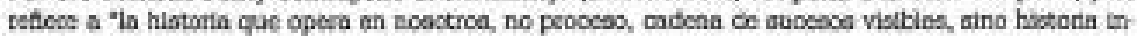

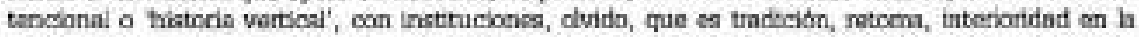

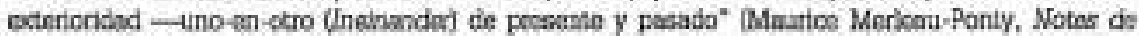
Cours at Caidge de Francr, 1958-1969 et 2960-1961, Paris, Gellimard, 1996, p. 80].

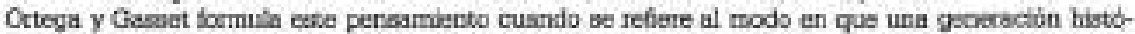

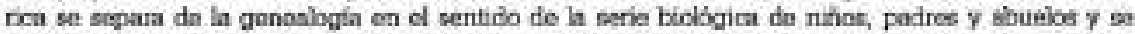
convierte en un "escorno do la historia uriversal" boeb Ottega y Guses, Chus Cumpletas, vol. V,

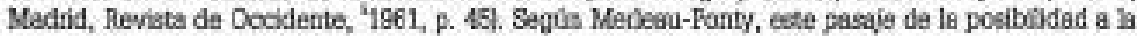

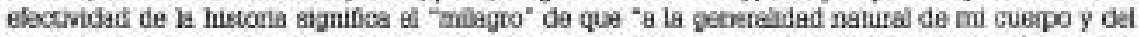

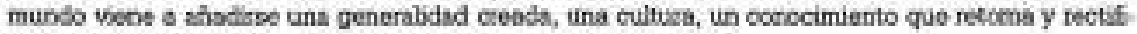

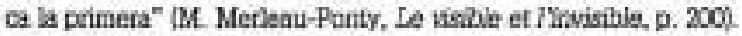


mediante una actividad operante por la subjetivided humana comunalizada en sus múltiples relaciones reciprocas" (hiua [X, 410). De acuerdo con los antes mencionados conceptos de Heidegger podriamos también decit que, en el pasaje del primer predarse al segundo, la prolongación entre el nacimiento y la muerte se marifiesta como el extenderse sobre los cambiantes portadores maduros de validez, la movildad de la adquisición se determina a partir de este proiongarse ampaiado, y la perduración se eleva a una recepcion bajo in guia de metas permanentes y supracrdo. nadas.

4.2. Husser analiza las tradiciones desde el punto de vista de las subjetividades unitarias de orden superior y las objetividades culturales, es docir, del bado noesion y el lado noematico: "La historia' es historia de la humanided o de una humanidad [... J. Ella es eo pso también histocia de la cultura, asi como la historia de la cultura es a una historia de la humanided, en plena concreción." (Hua XIV, 207; cf. IV, 360; VIII, 298; IX, 113).

4.2.1. En la medida cn que $3 c$ constituye cocno "un ente duradero", toda personalidad de ooden superior tiene oomo nosotros habitual su "habitualidad social" "Hua $\mathrm{XV}, 461,479)$. Respecto de estas adquisiciones permanentes de la experiencia intersubjetive pasada cabe destacar ante todo dos cosas. Por un lado hay una adquisición perclurable que contiente un predelineamiento en el antido del mundo ya constituido, y pot ol ctro lado un impulso perdurable que incluye un predelineamiento en el sentido de un mundo proyectado. Bn el segundo caso se inatituye una habitualidad de intereses que se presenta no solo como una cierta adquisición, sino también como una ocientación de la voluntad que determina el estilo de vida. Se trata de "un interés habitual que abarca incluso como 'interés vital' el horizonte abierto de toda la vida individual* (Ms B III 6, 2a). Los intereses se separan de lo meramente adquirído en razón de que no sclo relejan una sedimentación pasada síno que apuntan a un cumplimiento futuro. Para Hussen, pues, se distinguen "los auténticos intereses, los de los proyectos curaderos, de las metas 7 sistemes de metas, de las metas vitales (die eigentlichen Interessen, die der dauemden Vorhaben, der Zwecke und Zwecksysteme, der Labenszwacke)" de los "intereses alcanzados (erledigte Interessen)" (Ms C 3 V, 66b), es dectr, de aquellos que tuvieton un resultado favorabie y proporciosaron una posesión permanente. Porque estan enlazados con metas, los intereses autenticos se crientan como "hábito de la Lbertad" (Ms A. VI 28, 98a) ante todo hacia el futuro. Que el predarse sea también un planteamiento de tareas, se puede advartir corresativamente en un doble sentido ded horizonte historico que cocresponde tanio a los actos y las habitualdades adquirldas cono a los intereses y las metas que se han de efectivizar, es decir, tanto a lo alcanzado como a lo que se ha de alcanzar:

"El rmundo ya existente - el universo de bo alcanzado en cada caso. Fero tenemoe ura doble habitualidad: la habitualdad do lo ya existente y la habitualdad de las metas que alin guran y meras vitales que nos guian cumo peranas humanas y de un modo auténticemente humano, como hocizontes de metas, marcos para sisternas de scciones futurss" (Ms C $3 \mathrm{~V}, 66 \mathrm{~s})$. 
Según Husserl, los intereses juegan un papel importante en el enlace del horizonte de pasado con el horizonte de futuro porque "un pasado 'abarcable' y un futuro anticipado" son mediados "en torno de la fuente del ahora viviente" en razón de que los intereses actuales determinan "un particular horizonte de pasado y futuro como horizonte de intereses" (Hua XV, 397). Mucho recuerda aquí al problema, puesto de relieve por Ricoeur, sobre la coordinación del espacio de experiencia y el horizonte de espera. ${ }^{17}$ Advertimos ya en Husserl importantes anticipaciones que conciernen tanto a la dialéctica de espera y experiencia como a la dialéctica subordinada de la eficacia de la historia y el ser afectado. Por otro lado, el predarse en el segundo sentido corre paralelo con el segundo de los tres conceptos incluidos por Ricoeur bajo el título de tradición, esto es, con las diferentes tradiciones como portadoras de sentido de los contenidos transmitidos.

4.2.2. La historia es también historia de los objetos culturales, que son la encarnación y expresión de un sentido espiritual. Por eso se dirigen a sujetos personales y son los puntos de irradiación de efectos espirituales "sobre generaciones siempre nuevas en el marco de la continuidad histórica" (Hua XXVI, 21). Nuestra recomprensión de un producto del espíritu puede ser completamente exterior y tener el carácter de una mera indicación vacía: "Por la inercia de la naturaleza humana basta al hombre en un muy amplio ámbito una comprensión simbólica, sea plenamente vacía, sea intuitiva (efectivamente postproductiva) solo en una medida muy pequeña" (Hua XXVII, 110). Esto significa que los objetos culturales son "captados de un modo simplemente aperceptivo y tratados operativamente sobre el fundamento de la corporalización sensible, por ejemplo, por medio del lenguaje y la escritura" (Hua VI, 23). En la medida en que el sentido espiritual permanece en la indeterminación, es necesario un "desvelamiento histórico" (Hua VI, 379) por medio de la distinción y la aclaración. La distinción no es otra cosa que la explicitación del sentido-meta. Y el pasaje de la distinción a la aclaración coincide con el pasaje de la comprensión segunda del sentido a la experiencia de los fines. Así, las formaciones culturales "son experienciables de múltiples maneras en el modo de la recomprensión interpretante y eventualmente también experienciables en una validez general de fines, como conformidades a fines en el sentido de un sistema válido de fines" (Hua IX, 117 s.; vgl. XXIX, 375). Ello significa que la recomprensión ha de ponerse en el lugar de una aspiración orientada por fines si quiere alcanzar claridad. Y la claridad significa "comprensión intuitiva del fin general" (Hua IX, 113). Esto exige también una comprensión de las correspondientes circunstancias, de la situación del entorno temporal, y del ulterior sistema de fines de la vida de la comunidad.

4.3. La tierra se muestra en este nivel como el territorio total que comprende en sí todos los territorios parciales. A cada una de las antes mencionadas formas de la generatividad es inherente un territorio particular como mundo circundante territorial o campo vital geográfico con casas, caminos, campos, etc. Como espa- 
cio cultural, el territorio tiene su ámbito externo y su capa infericr de mera naturaleza, pero es siempre campo de la praxis y mundo de bienes: "Cada humanidad no tiene abstractamente su naturaleza, sino naturaleza como núcleo de su cultura. Cada une tiene su naturaleza humanizada como suelo permanente, configurada en el marco territorial en cultura de cosas, en cosas con sentido espiritual y pot ende histórico" (Ms B I 15 II. Tr. 9). Bs digno de atención que Husserl asigne al "nosotros" que ocupa un territorio una "corporalidad colectiva (kclloktive Leiblichkeit)" (Ms B I 15 IV, Tr. 4), porque el territorio detemina para cada uno un lugar y una arientación. ${ }^{\text {to }}$

Cadia grupo histótico que vive en un territcrio tiene su raptesentación del mundo. La tierra es originariarnente "el suelo experiencial para todos los cuerpos en la génesis experiencial de nuestra representación del mundo" "En tanto tepresentedo el mundo ee construye ccrno un "proyecto (Entwurf" que no se limita a las posibilidades inmediatas del entorno: "[... puedo construir una 'repreaentación del mundo' como proyecto, un mundo representado 'de antemano' en sus pceiliilidades" QMs A VII 1, Tr. 9). Las posibilidedes pueden ser establecidas de nuevo de diforente manera, porque el mundo circundante, que está en la base de la representeción del mundo, se transforma según los respoctivos intereses prácticos. Lo cual significa que cada humanidad histórica unitaria, del mismo modo que cada hombre singular, es portadora de una repcesentación del mundo. Su estructura remite en cada caso al mundo actual de intereses. Asi como la explicitación de una cosa sangular produce una representación de su sar-asi, las cornundades configuran regresentaciones del mundo a fin de poner en claro lo que está impitcado en el fuyente y desvanectente horizonie histórico.

\section{La segunda historicidad}

El paso siguiente que se debe dar con Husserl consiste en la caracterizacson de la segunda historicidad. Se trata aqul de una esfera totalmente nueva de problemss, en que, puesto que se relacionan con una destacada teleologia, las dimensioneg de la generatividad, la tradición y el tecritorio tienen un nuevo sentido: ya no se puede hablar de metas finitas. Bntre las tradiciones sobresale aquella cue introduce un tipo esencialmente nuevo de generatividad espiritual a partir de un vinculo segùn metos L... en la historicidad general humana" (Ms K III 3, 38a). Frente a las otras tradiciones, Husserl distingue "la nueva tradicionalidad de la teorja" (Hua XXIX, 15) como "dosarrollo de la totalidad monádico-racional historia en sentido estricto" (Hua XV, 669; cf. VIII, 241). Las ineas fundamentales de esta segunda historicidad, que aparecen ante todo en los articulos de Kaizo y en la

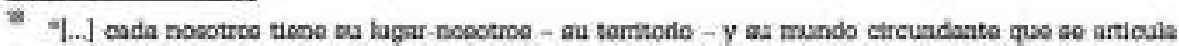

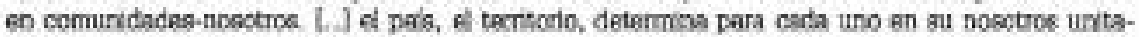

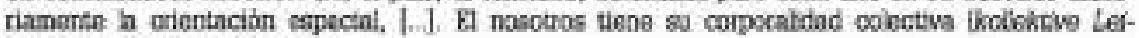
blichkeity (Ass B 15 IV, Ir. 4 .

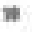

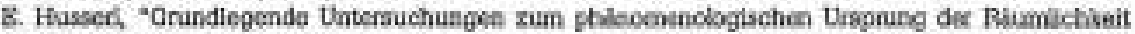
der Natur, p. 3ah
} 
Conferencia de Viena, no necesitan ser repetidos aquí. Solo se han de esbozar sucintamente las formas fundamentales en que se muestran las tres dimensiones de la relación con el otro, el tiempo y el mundo.

5.1. La teoria permite surgir una comunalización de nueva índole, porque su tendencia a la extensión carece de límites en la medida en que las formaciones ideales pueden ser reasumidas sin más por cada hombre racional. Con ello se introduce una unidad instituida y universal en el nivel espiritual superior de la generatividad científica y filosófica, que es planteada como telos para toda la humanidad y tiene "el significado de la progresiva transformación de toda la humanidad" (Hua VI, 322). Por eso aparece una forma universal de generatividad frente a las formas y niveles limitados.

5.2. Puesto que constituye para Husserl el punto de vista decisivo para los niveles de la historia, la teoría desempeña el papel de "la" tradición en el sentido del tercer concepto de tradición destacado por Ricoeur como modo preeminente de transmisión de sentidos. Los hombres se mueven en un "sinnúmero de tradiciones" (Hua VI, 366) y cada tradición se remite a ideas comunes, porque las ideas se conectan indisolublemente con las metas. Pues la reiterabilidad de la actividad según metas es la condición de la idea, y esta emerge a partir de una extrapolación que se funda en la progresiva explicitación de los horizontes.

Aquí se han de distinguir las "ideas de infinitud" inherentes a la generatividad racional espiritual de las "ideas de finitud" inherentes a la humanidad prefilosófica. En estas solo se da un horizonte futuro abierto, en aquellas se da concomitantemente también la posibilidad de una repetición de lo mismo, la generación de idealidades de orden superior y el acercamiento a la verdad en sí como un polo infinitamente lejano. Las ideas de infinitud presuponen formaciones ideales que conforman "el fondo de premisas para un horizonte de tareas infinito como unidad de una tarea omniabarcadora" (Hua VI, 324). Junto a las verdaderas ideas infinitas hay ideas que solo tienen "los caracteres análogos de la infinitud". Aun cuando permanecen "fuera de la esfera filosófico-científica", reciben esta modalidad peculiar a partir de las ideas de la filosofia y la ciencia, cuando son "formadas de nuevo en el espíritu de la idealidad filosófica" (Hua VI, 324 s., 335).

5.3. Finalmente, debemos subrayar la intención de la teoría de conocer el "mundo verdadero en sí" (cf. Hua XV, 215 n.) frente a las diferentes representaciones del mundo.

${ }^{20}$ Sobre la relación entre la tradicionalidad y el concepto de "interés", y la realización del telos racional como verdadera satisfacción de los intereses, cf. Hans Rainer Sepp, Praxis und Theoria. Husserls transzendental-phänomenologische Rekonstruktion des Lebens, Freiburg / München, Karl Alber, 1997.

21 Cf. Klaus Held, "Heimwelt, Fremdwelt, und die eine Welt", Phänomenologische Forschungen, 24/25, 1991, pp. 324-336. 


\section{Horizonticidad y temporalidad}

6.1. La historia efectiva se ajusta a una forma de la generatividad, un territocio $y$ una tradición, $y$ estas tres dimensiones pueden ser sometidas a un doble proceso de indeterminación y deteminación. Una indagación tetrospectiva busca un núcleo del mundo que se prepara y se extrae abstractamente" (Hua VI, 136), y conduce en dos pasos a la trieda protogeneratividad-tradicionalización-tierra y a la triada de las implicaciones. Nos wolvernos retrospectivamente desde la efectividad de la historia, a través de las condiciones previas para la efectivización, a ha posibilidad de las meras implicaciones. Así se pone de relieve la indeterminactón de lo que se denomina implicación frente a la determinación de las condiciones de la efectivización, la uiterior determinación de los diferentes hilos de los desanollos históricos efectivos, y la señaleda determinación que Husserl asigna a la segunda historicidad. Ceda uno de los niveles en el avance contrapuesto se apoya sobre el predelineamiento anticipador del anterior a fin de alcanzar el mismo, como dice Hussetl, un "progreso en la concrecion" (Hua XV, 138 n.).

A partir del nivel de la efectividad, el entramado y contraste de determinación e indeterminación recibe un nuevo sentido, que nos renite a los "honizontes de cognoscibilidad e incogmoscibulidad " (Hua XV, 431) en relacićn con generaciones culturales, tradiciones y territorics. En lo que concteme a este enriquecimiento se pueden distinguir das aspectos. Pues las hetencias y las metas familiares so destacan frente a la latencia de la propia tradicsion y la incognoscibilidad de las tradiciones extrañas. Por un lado, el presente histórico de cada tradición implica el pasado de la cultura "en una generalidad indeterminada, pero estructuralmente determinada" (Hua VI, 379), con lo cual Husserl distingue entre historicidad latente y explicita. Por otzo lado, el contraste entre el mundo familiar con su historia interior y el mundo extraho oon su historia exterior significa la "diferencia de oomprensión entre lo familiar-comprensible y lo extraño-inosmprensible" (Hua XXIX, 42; vgl. 5,63 8.).

En el horizonte histórico tenemos que ver con un juego recigroco de dos momentos, de los cuales uno desempeña el papel de hilo conductor y el otro el papel de la incitación para ir más allá. Bste entramado de determinecićn e indeterminación es lo propio de la borizonticidad, que exhibe una doble significacion en relación con el pasedo y el futuro. Por un Jado, la implicación en el modo del horizonte no se ha de entender como una timitada remisión más allá de lo dado en cada caso. Puesto que una estructura de infinitud queda planteada de esta manera, el horizonte histónico puede ser caracterizado bajo la representación de la ides en

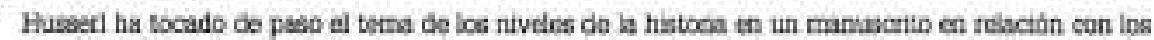

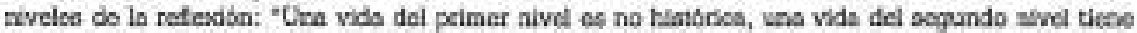

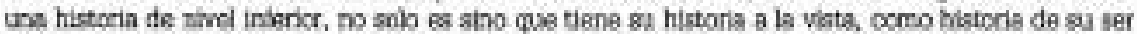
y de su mundo cercundante hist6000", a una coo ella. En el tercer nived la tustoria obtiens su sentsdo aheobuto |. I. Se necesila por cketto de algun complomento a fin de llenas buocos que han que-

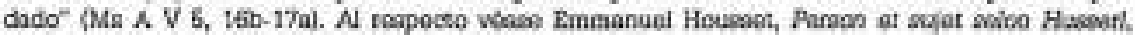
Para, Presese Unimersitaires de France, 1997, Dp. 194-205.
} 
sentido kantiano. Por otro lado, el horizonte no se ha de entender meramente como una remisión indeterminada más allá de lo dado hacia algo externo. De la circunstancia de que está dada una estructura de la determinación o de la adquisición, se sigue que el horizonte histórico puede ser considerado bajo la imagen del suelo. Así, horizonticidad, idea y suelo son los rasgos fundamentales del concepto husserliano de mundo histórico. El horizonte se muestra como indeterminado o como determinado, esto es "como constante horizonte 'suelo" (Hua XXIX, 302). El suelo se muestra como protosuelo (Urboden) o "como adquisición, como suelo familiar" (Ms A VII 20, 9b). Y las ideas pueden ser finitas dentro de la primera historicidad o infinitas dentro de la segunda historicidad. Cuando se distinguen de esa manera niveles y dimensiones en la teoría husserliana de la historia, resulta el siguiente esquema:

predarse I

posibilidad de la historia predarse II

efectividad de la historia

$\begin{array}{cccc}\text { formas de la } & \text { condiciones de la } & \text { historicidad } & \text { historicidad } \\ \text { implicación } & \text { efectivización } & \text { I } & \text { II }\end{array}$

otros

protogeneratividad

formas y niveles

de la generatividad

generatividad

universal

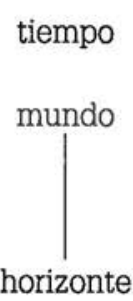

tradicionalidad

tradiciones

"la" tradición
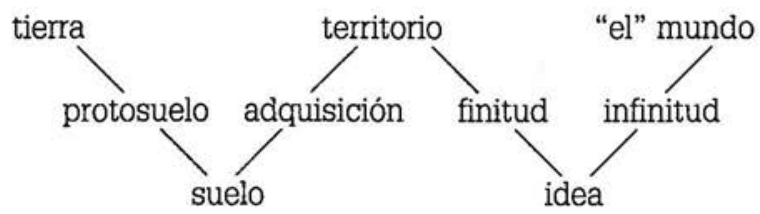

\section{$\leftarrow$ Indeterminación \\ Determinación $\rightarrow$}

6.2. Estas consideraciones posibilitan una contribución al desarrollo del problema de la unidad o de la pluralidad de los tiempos histónicos, que, como ha observado Merleau-Ponty, debe ser tratado "por medio de un retorno a la idea de horizonte". ${ }^{23}$ De acuerdo con la anterior explicitación del horizonte histónico se pueden separar claramente uno de otro cuatro diferentes conceptos de tiempo, que se superponen uno con otro y de los que cada uno es la expresión de un modo de unidad de la historia:

${ }_{23}$ M. Merleau-Ponty, Le visible et l'invisible, p. 239. 
1. el tiempo como protencionalidad instintiva originaria /- procontstorla);

2. el tiempo como una formación espiritual que se mantiene por la reactivacion de una proinstitución (- primera histoticidad, tiempo de los objetos culturales y de las subjetividades de crden superior);

3. amnitemporalidad como modo de la temporalidad ( - eventualmente, segunda historicidad);

4. tsempo como horizonticidad indeterminada (- mera forma vacia).

6.2.1. En el nivel de las condiciones previas para la efectividad se depliega, como ya se mencionó, siempre una protopeneratividad, que, sobre el suelo de la unica tierra, fundamenta una unidad de la historia que no es instituida, es decir, no proviene de actos. Asi, el tiempo se conecta en este nivel con la historicidad protencional ociginaria. Husserl slude a "una intencionalidad instintiva universal que configura unitariamente cada presente primigerio como constante temporalización y continúa impulsando concretamente de presente en presente [...]" (Hua XV, 595). Asi tiane una función vinculadora scbre la cual se asiente un entramado temporal de los diversos cursos singulares.

En lo que concierne a la tierra como sublo oxiginario de una protohistoria, Husserl caracteriza al tiempo histócico como "necesariamente en coincidencia con el tiempo de la naturaleza (Hua XXIX, 83). Esta es la forma de unidad de la totalidad de los cuerpos, en la que participen todos los hombres por su corporalidad. Puesto que la estructura naturaleza pertenece a la estructura dol mundo, "todas las cosas tienen un lugar en el tiempo de la naturaleze, el tiampo espactal, y todo Io no natural en ellas st lugar anexo (seine annektierte Stallung) en el mismo" (Ms $K$ III $6,146 a$ ). Ast, el cambio del dia y la noche y el cembio de las estaciones proporcionan las primeras medidas de tiempo para un tiempo común." A ello se añade que los trechoa tamporales en el pasado y el futuro están límitadce por el nacimiento y la muerte. Pero el tiempo histórico, según Hussari, no está dado aún con el tiempo de la naturaleza, porque presupone un nexo de todos los sujetce, Y $\mathrm{co-}$ mo forma de concreción de este nexo el tiempo histórico es "un tienspo de orden superior" (Hua XV 340). Teniendo en cuenta astas relaciones de fundamentación, Huserl puede afirmar: "La eatructura de realidad del mundo circundante práctico, del mundo de la vida, no es otra cose que la estructura natural on seatido amplio" QMs A VII $14,5 b)$.

6.2.2. Fin relación con el hocizonte como suelo adquirido e idea proyectada, la unidad de la historia significa la reforencia a una institución injicial y a una institación final La historia es ocncebida por Husent "a partir de la unidad y fuerza impulgiva de la tarea" (Hua VI, 72). Una intencionalidad intareubjetiva, que depende de las posiciones de metas y de loe cumplimiectos relativos, posibilita "una unidad de la vida ristirica que onlaza presente con presente, loe ahora vivientes con los ya no

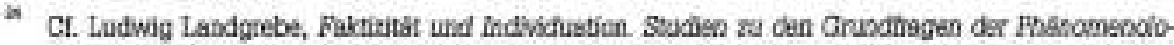
gie, Hsmburg, Menes, 1982, D. 51; - D uempo coentin tepose sobte of 'lado napata' de la sutjerivdad, qoo tiene al mismo uns función constituyente". Cf. $K$. Heid, "La experianeia gonerativa del

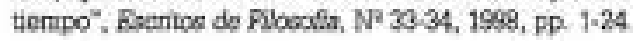


vivientes" (Ms K III 3, 38a). Toda unidad dd espiritu histónico es "corralato de la unidad del operar, que paea a través de una plusalidad de peraonas, las poetenores ejarcen impotis en la vida de las anteriores, en la medida en quo recomprenden sus operaciones como operaciones (...)" (Hua XIV, 198). De aqui resrlta que nas encontramce, en unjón con todos los nexos generativos, "en $\&$ curso unitario de una historictdad" (Hua VI, 256). Se trate en cada caso de una unidad que une la tnatitución originaria can nuevas ingtitucicnes bejo la guia de determinadoe intercees reguladores. Si mi preestre y sus horizontes temporales se reculve de este modo con el presente de loe otroe y sus horizantes, se constituye "un a la vez temporal del tiempo supcamonádico o intermonádico de crden superior" (Hua XV, 343). Pce eso Husseet subcaya que el tiernpo es "una formación erpiritual dal hombre (ain geistigos Gabilde des Merishenen) (Ms A V 24, Tr. 18; of. Hua XV, 605).

Las personalidades comunallaadas como una personalidad de orden superior configuran une "uridad de duracibn"n" y tienen un saber chradero de eata unidad. Aff surgen enlacos personales "do funcionabiolad relativamente permanente" y "formas o modos permanentes de vica operante efectuante" (Hua XIV, 206) $Y$ con ello "sc onnstituye el tiempo comunitario y loe modice del darse subjetivo de eate tiempo, del darse subjetwo comunitario" (Hua XIV, 221). En esta termparalidad eetán inclusdas todus las adquisiciones y tambien las cosas irrelevantes pera los inteteeses humanos, Puesto que el hombre comunalizado tiene su "lugart en un copsacio social, asi como las realidades fisicas eatán localizadas en la única naturaleza fissca, el tiempo histórico se muestra corno un "onálogo de la espacioternporalidad en sentido habitual" (Ma C 36 III, 25a), esto es, en el sentido de la nasuraleza abetracta. De este modo Hussecl enaliza "le espaciotemporalidad generativa constituida en comeleción ocn la temporaLidad personal generativa (intersubjetivamente inmanente) [... I" (Ms A V 16, 9b).

Junto con la coíncidencis de los tiempos inmanentes, que se encuentran coimplicados por la comunalización del único tiempo (ct. Hua XV, 334 ss., 633 ss.), Hussed destaca dos rasgos fundamentales del tempo históxico. For un lado, respecso del futuro, se produce una 'tension temporal entre el 'yo quitaro' instituyents y la ejecucion, o sea, el hecho" (Hua XXXX, $366 \mathrm{n}$ ), esto es, entre el querer y la realización de sos intereses. Por eso Hussert no explica nuestra adopción de la tradicion meramente como la recomprension de la institucion cringara, sino tam-

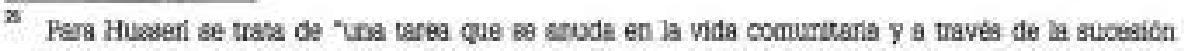
de las genecactones, de los tiempos hiathriocs" (Hua XCXIX, 304). Husserl se refiare tambucin a a " $u$ -

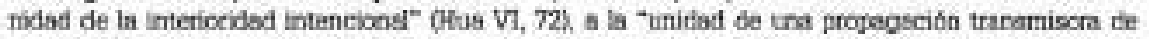

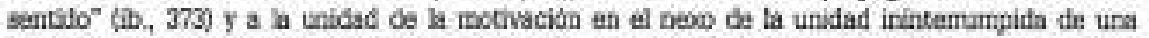

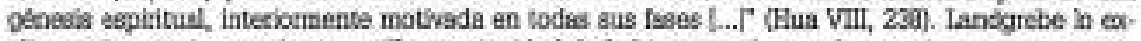

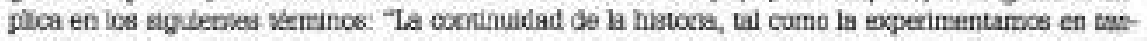

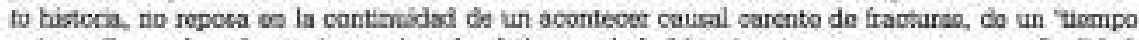

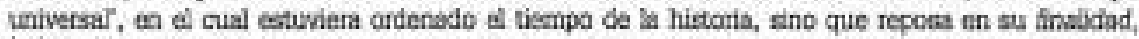

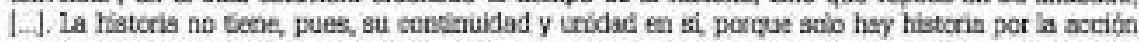

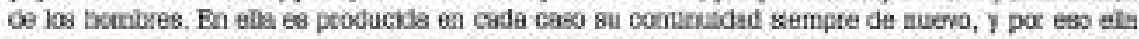

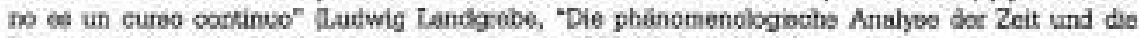

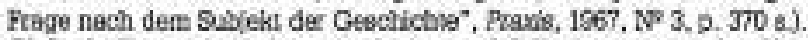

ct. Lazlo Tergelyi, "Lebenegeschichte und Selberkonstitution beil Hasserl", Huzsed Stindies, 13, N" 2, 2997, pp. 155-167. 
bién como coinstitución y coefectuación de vigencias Lo cual significa una "decisión por un horizonte temporal" (ib. 371), que conduce a que emerjan diferentes ámbitos temporales, Se debe observar aquil que toda praxis efectiva de quienes sustentan la vigencia de una tradición y obran en el mundo no solo depende de las circunstancies y debe superar los obstículos, sino que también puede convertirse "en una pasividad "ciega"' que conduce al "fracaso del proyscto" (ib. 383). Por otro lado, respecto del pasado, es inherente al tiempo histórico el tecuerdo de acontecimientos que el sujeto no ha vivido efectivamente. No ha habido una experiencia propia de lo que se recuerda comunitarlamente en una situación que no deja de exhibir una clerta analogia con el propio pasado experienciado que se ha olvidado defintivamente. De ahi que la explicitación del horizonte histoxico exdia una rememoracion en el "como si" en conexión con un peculiar tipo de impatia. Hussarl habla del "modo de la impatia generativa (Modus generstiver Binfühingl que exige un encedenamiento de impatias intencionalmente implicadas, efectivas y posibles" (Ms A V 16, 12b; of Hua XXIX, 343 s. zf $^{77}$

Importante para la caracterización del tiempo histórico es también el análisis de la temporalidad del objeto cultural. ${ }^{28}$ Pues la posición de su realidad corporal en el mundo real asigna al sentido espiritual "un modo de la duracico temporal" y "algo as como efecto en el mundo" (Hua $\mathrm{LX}, 117$ ). Puesto que ge convierten en puntos perdurables de irradiación de efectos espirituales, las formacionos cuiturales motrvan el deopliegue de una temporalidad espiritualmente formada. En esta función pueden tener "una exiatencia paajora en el mundo", o, cuando an imperecederas, "una temporolidad totalmente distinta" (Hua VI, 323). Esto dopende de si tienen una relación con la existencia coeno los objotos de uso o las obras arquitectónicas, o no como los objetos espiritueles ideales (ct. Hua IV, 238).

6.2.3. Lo que se delinea aquí con esta otra temporalidad es una ausencia de temporalidad, que ya aparece en la primera historicidad y se relaciona estrechamente con la telealogia peculiar de la segunda historicidad. El punto de vista rector es el de la repetiblidad idéntica, puesto que posiblita la producción, inherente a la tearla, de nuevas formactones sobre la base de las precedentes en un proceso al infinito. Lo decisivo de las idealidades reside en que podernos volver a ellas una. y otra vez porque tjenen la 'forma fundemental del 'y ast sucasivamente', de la 'infinitud' iterativa" (Hua XVII, 195). Husserl subraya que esta intemporalided, que se muestra como supratemporalidad u omnitemporalidad, no es otra cosa que "un modo de la temporalidad".

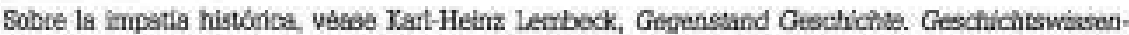

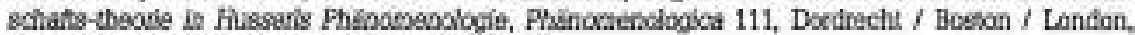
Kouwer Academic Publistees, 1988 , pp. 192-228.

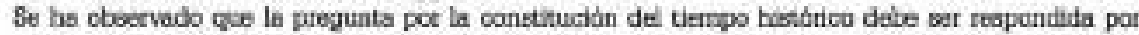
medin de uns investigación sobre el abjeto histárico parcese en portble que elle pasga de manifiesto

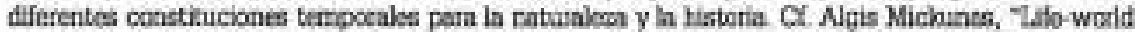
and Fistscy", en Burt C. Hopisins (ed.) Hessed in Contempcrary Context. Prosoecte and Projects far

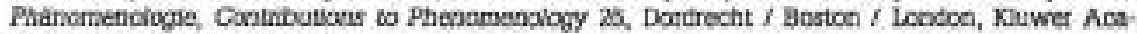
domio Publathocs, 1997, p. 204

EAmund Hurset, Erfahrung und Urand, Hamburg, Classen, '1964, p. 313. 
Ante todo se debe tener en cuenta que las formaciones ideales son reproducibles "en cualquier luggar temporal" (Hua I, 156) de modo que su irradiación de efectos es ilimitada. Si miramos la cuestion más de cerca, debemos distinguir niveles. Bn el estadio superior se encuentran las idealidades de mayor grado de identidad como el teorema de Pitagoras o un fllosofema, que se corpcralizan en multiples realizaciones en el mundo por medio del lenguaje, pero aparecen numo ncamente identiccs en estos lugares espaciotemporaies, porqque "esta localización no las individualiza efectivamente" (B III 5, Tr. 7). Lo mismo sucede con otras formaciones literarias como el Fausto de Goethe o la Heroica de Beethoven, que se corporalizan en libros, partituras, etc., y pueden ser reactivadas como las mismas, si bien no tiene como aquellas una orientacion teleologica hacia metas infinitas. En un estadio inferior se encuentran idealidades como la Madona de Rafael, que son en principio repotibles, aun cuando "tienen fócticamente una única mundanidad y fácticamente no son repetibles en una identidad suficiente (la del pleno contenido ideal)" (b. Tr. 8). Asimismo, Husserl asigna una idealidad a una constitución estatal en tanto es recomprensible e jdentificable por diferentes persones (cf. 1b.; Hua IV, 243). Por último, no se he de pasar por alto que hay un sentido en que la repetibilided no es extrahia a las objetcs culturales perecedercos. Husserl observa que el hombre impregna en las cosas estructuras de fines "por medio de las cuales ellas se convierten para él en instrumentos, en adquisiciones útiles, para ser utilizadas repetidamente, "siempre de nuevo"' (Ms A V 24, Tr. 15)."

6.2.4. Finalmente queda por analizar b horizonticidad indeteminada en relación con el tiempo histótico. Con ello retomamos al punto de partida, esto es, a las condiciones de posibilidad. Nás allá del horizonte cotidiano de intereses ee encuentra, seguin Husserl, el "horizonte de mundo apráctioo", al que caracteriza como "un horizonte de mundo que contiene otras humanidades y sus mundos de la vida y traspasa finalmente en un horizonte de munto "vacio"' (Hua XV, 141; cf. 232, 411). Todos los mundos de la vida con sus diferentes ámbitos temporales son abarcados por este horizonte vacio: "Tambiabn el concepto de horizonte se escinde. Horizonte de la situación - se evoca un interes de la vida como horizonte, en al que se mantienen las actividedes momentáneas; ovecado es de otra manera todo el horizonte del mundo en la medida en que el mundo siempre está ahi" (Ms C 3 V, 66a). Esta eeciaión de la horizonticidad corre paralela con la diferenciación de las representaciones del mundo de las difarentes tzadiciones por un lado y del horizonte del mundo con un horizonte temporal vacio por el otro. Se debe tener en vista el único mundo como horizonte universal al se quieren aclarar las relaciones entre las diferentes y limitadas tradiciones con sus repressntaciones del mundo. A pesar del conflicto de determinados horizontes unos con otros, la indeterminación del horizonte deja abjerta "la posibilidad de una adopción (Ubermahme) concot-

* Sobo la idsalidad omnitenpocal del scotido del objoto cultural y h realizacion concocta atada al terngo, wase X.-H. Lembock, op cat., P. $178 \mathrm{~s}$ 
dante bajo corrección" (Ms K III 2, 71b). ${ }^{31} \mathrm{Si}$ la representación del mundo y el horizonte del mundo no llegan a diferenciarse, se cae en una incompatibilidad, que impide toda fusión de horizontes entre las diferentes humanidades. Por eso lo peculiar del horizonte del mundo reside en el modo en que posibilita los pasajes entre diferentes tradiciones y representaciones del mundo. Estos enlaces presuponen tanto la indeterminación abierta del horizonte del mundo, que los mantiene en marcha, como la determinación de un suelo terrestre y una única generatividad: "[...] así reside aquí la validez previa de la capacidad de los hombres como capacidad de esta humanidad de poder entenderse y armonizar" (Ms K III 6, 145b).

Cuando es considerado solo como horizonte, el mundo ha de ser caracterizado como mundo "sin orden temporal, como capacidad de la determinación temporal (ohne Zeitordnung, als Vermöglichkeit von Zeitbestimmung)" (Ms A V 19, Tr. 49). Pero como una formación espiritual, el tiempo asume dentro de una representación del mundo "la forma del tiempo ordenado y del tiempo medido (die Form der geordneten und der gemessenen Zeit)" (Ms A V 24, Tr. 18). Con ello surge el problema de aclarar hasta dónde se puede hablar con sentido de la dimensión temporal respecto del horizonte histórico vacío. En relación con la determinación de esta dimensión como una forma de horizonte abstracta entre la representación intuitiva del mundo con su tiempo ordenado y la mera indeterminación, Husserl escribe:

"La forma vacía del tiempo histórico, del horizonte de los 'tiempos' con el centro vivientemente fluente del presente histórico, los tiempos mismos formas vacias de los presentes sidos, en las manera subjetivas del hacer-se-retroceder fluentemente, $\mathrm{y}$, con este horizonte de retrodesplazamiento, que es él mismo vacío. [...] Pero el horizonte histórico como esta forma vacía es algo a lo cual puedo prestar atención en la representación del mundo, que siempre transcurre 'de un modo concretamente intuitivo' y continuamente en cambio modal" (Ms K III 10, 13a).

Ante todo es necesario considerar cómo los modos de la experiencia del tiempo, o sea, de la "modalización del tiempo (Zeitmodalisierung)" (cf. K III 6, 146a) se enlazan con la precomprensión de las estructuras formales y la articulación del tiempo, ${ }^{32}$ a fin de poner de manifiesto una copertenencia a una forma. Así como los tiempos particulares de un sujeto se ordenan bajo una forma total, los diferentes tiempos vitales pueden ser ordenados bajo un tiempo total. Cada hombre tiene "diferentes presentes comunitarios", y esto depende de que él en cada caso participa en un horizonte distinto de intereses predominantes. Sus horizontes de intereses pueden ser aquellos de una organización, su familia, su pueblo, etc., cuyos correspondientes tiempos se enlazan en un tiempo histórico universal como su forma total, de modo que ellos como contenidos temporales aparecen en la

${ }^{31}$ Sobre esta posibilidad véase Ludwig Landgrebe, "Lebenswelt und Geschichtlichkeit des menschlichen Daseins", en Bernhard Waldenfels, Jan M. Broekman y Ante Pazanin, Phänomenologie und Marxismus. 2. Praktische Philosophie, Frankfurt a.M., Suhrkamp, 1977, pp. 41-42.

32

Sobre la relación anterior-posterior, cf. Elisabeth Ströker, Phänomenologische Studien, Frankfurt a.

M., Vittorio Klostermann, 1987, p. 193 s. 
forma total según la simulaneidad o la suceston temporal, y se destacan de un horizonte de historia desconccida. Hussetl da a esta concepción la siguiente formulación: "Cada mundo da presente de estos diferentes modos se encuentra empero en un munto, en un horizonte externo pero infinito, que traspasa todo to conodido corno horizonte de los pasados y futuros desconocidos" (Ms K III 6, 144b).

Para terminar pongamos de releve que el horzonte del mundo no solo mediatiza los vinculos entre tradiciones históricas con sus diferentes representaciones históncas, sino que proporciona la mcitación para ir mas alla de los limites de una representacion del mundo o un horizonte de intereses. Su estructura de indetermipación se asocia con posibilidades tneditas de sentido que se sustraen a todo marco dado de deteminación. Lo que es valido para el mundo en Merleau-Ponty. esto es, que es 'absolutamente diferente de lo 'representado', a saber, como Ser vertical, al que ninguna de las 'representaciones' agota y que todes 'alcanzan', $[\ldots . .]^{*}$, se revela también vigente en Husserl para el horizonte del mundo.

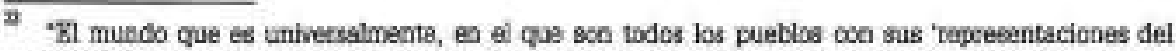
mumdo', con sus en cadi caso proplas 'repuesentaciones del nubdo', sus botizontictfades - vigenclas gesetales del mundo, en el casi para cads puebto ea el nodo del horizoate' al metros sont to-

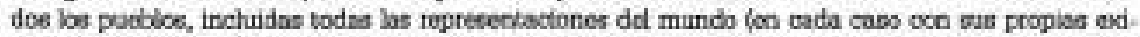

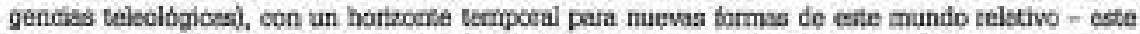
como haxizoste abierto de pustibe cunocidas y desconocidos, ocientado a partir de cada puebio y

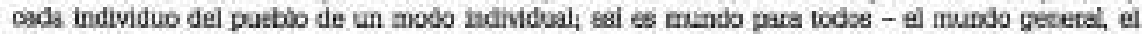
mundo paro y aimplo - el mundo en ol que la humanidad viwo - su mundo trietónco" 0 Ms K II 6 , 3118).

M. Merlasu-Ponty, Lo visibie et $I$ 'anvisibje, p. 305 . 